\title{
New Data and Modelling for Single Event Effects in the Stratospheric Radiation Environment
}

\author{
Alex Hands, Member, IEEE, Fan Lei, Member, IEEE, Keith Ryden, Member, IEEE, Clive Dyer, \\ Senior Member, IEEE, Craig Underwood, Member, IEEE, and Chris Mertens
}

\begin{abstract}
The upper atmosphere is a transition region between the neutron-dominated aviation environment and satellite environment where primary protons and ions dominate. We report high altitude balloon measurements and model results characterising this radiation environment for single event effects (SEE) in avionics. Our data, from the RaySure solid-state radiation monitor, reveal markedly different altitude profiles for low linear energy transfer (LET) and high LET energy depositions. We use models to show that the difference is caused by the influence of primary cosmic ray particles, which induce counts in RaySure via both direct and indirect ionization. Using the new Model of Atmospheric Ionizing Radiation Effects (MAIRE), we use particle fluxes and LET spectra to calculate single event upset (SEU) rates as a function of altitude from ground level to the edge of space at $100 \mathrm{~km}$ altitude. The results have implications for a variety of applications including high altitude space tourism flights, UAVs and missions to the Martian surface.
\end{abstract}

Index Terms-Single Event Effects, Stratosphere, High altitude radiation environment

\section{INTRODUCTION}

$\mathrm{T}$ HERE are many different types of radiation environment in which single event effects (SEE) can pose a threat to electronics in aerospace applications. In medium Earth orbit (MEO), geostationary orbit (GEO) and interplanetary space the hazard comes from protons, alpha particles and heavy ions in the galactic cosmic ray (GCR) background and solar particle events (SPEs). In low Earth orbit (LEO) there is the additional, and often dominant, factor of trapped protons in the South Atlantic anomaly (SAA). By contrast, the SEE threat in the atmosphere at aviation altitudes and at ground level comes primarily from secondary neutrons produced by collisions between primary protons and molecules in the upper atmosphere. This picture implicitly identifies a wide transition region between the troposphere and terrestrial-space boundary. This region, which for all intents and purposes is defined by the stratosphere, has not historically been one of interest to the radiation effects community. Little progress has been made

Manuscript received July $10^{\text {th }}, 2016$. This work was funded by the NASA Science Mission Directorate under the Hands-On Project Experience (HOPE)-4 opportunity.

A. Hands, K. Ryden and C. Underwood are with Surrey Space Centre, University of Surrey, Guildford, UK GU2 7JP. F. Lei is with RadMod in this area since the work of Tsao and Silberberg in the 1980s [1], although recently some work has been performed to directly measure and model SEE rates in the stratosphere [2] [3] [4]. However, with the advent of space tourism and the reinvigoration of interest in high altitude surveillance, communications and even passenger platforms (both aircraft and balloons in each case), it is important that this environment is studied and understood in a radiation effects context. In addition, this area of research has application to the Martian surface environment where the total atmospheric depth is approximately equivalent to the high-altitude terrestrial environment at around $25-30 \mathrm{~km}$ (approximately $10-20 \mathrm{~g} / \mathrm{cm}^{2}$, see [5]). In this paper we present data from a silicon-based detector flown on a high-altitude balloon experiment and we use these data to validate modelled atmospheric particle fluxes and to predict single event upset rates as a function of altitude.

\section{MEASUREMENTS}

The Radiation Dosimetry Experiment (RaD-X) was launched in September 2015 on a high-altitude balloon platform [6]. The flight, launched from the Columbia Scientific Balloon Facility in Fort Sumner, New Mexico, lasted for approximately 22 hours and achieved a peak altitude of approximately 125,000 feet $(38 \mathrm{~km})$. The geomagnetic cut-off rigidity at this location is $4 \mathrm{GV}$. The altitude profile and flight route map are shown in Figure 1 and Figure 2 respectively. Space weather conditions at the time of the flight were quiescent with no observed enhancement to the galactic cosmic ray (GCR) background flux. The RaD-X payload consisted primarily of a suite of radiation instruments, including the silicon-based RaySure detector [7]. RaySure uses a large-area PIN (p-i-n) diode to count particle interactions using fifteen logarithmicallybinned energy-deposition channels. The threshold linear energy transfer (LET) is given as a function of channel number in Figure 3. For ease of reference, in this paper we define channels 0 to 5 as low LET, channels 6 to 10 as medium LET and channels 11 to 15 as high LET.

Research, Camberley, UK, C. Dyer is with Surrey Space Centre and CSDRadConsultancy, Fleet, UK, Chris Mertens is with NASA Langley Research Center, Hampton, Virginia, USA. (Corresponding author telephone: +44 1483686262; e-mail: a.hands@surrey.ac.uk). 


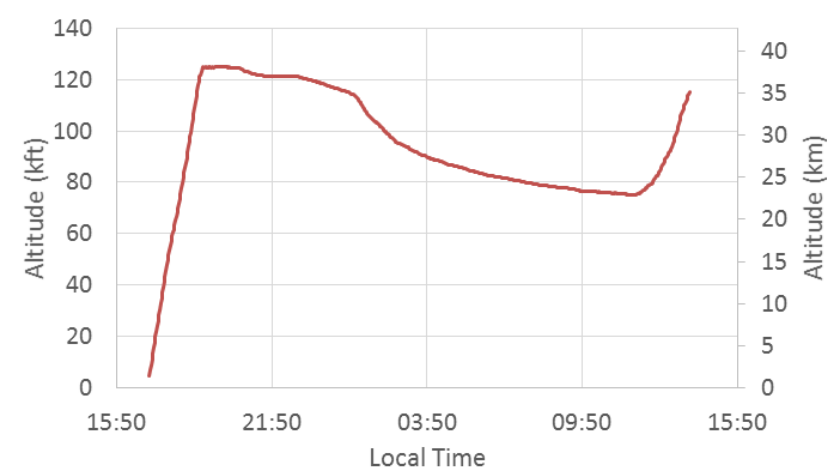

Figure 1: Altitude profile of RaD-X balloon flight on $25^{\text {th }}$ September 2015. The payload was launched from Fort Sumner, NM at approximately 1700 UT and landed 22 hours later a few miles outside of Roswell, NM.

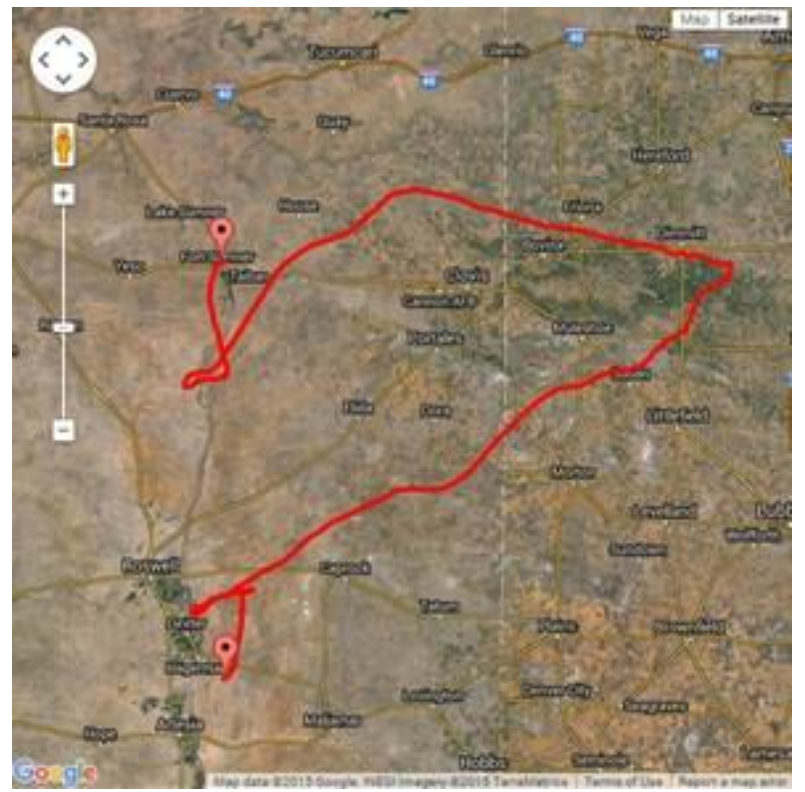

Figure 2: The route taken by the RaD-X balloon flight. The launch and landing sites at Fort Sumner and Roswell are identified by the top and bottom markers respectively.

The dynamic range allows for a variety of particle species to be detected in the mixed radiation field of the atmosphere, including direct ionization from protons, ions and the electromagnetic cascade (electrons and gamma), as well as indirect ionization from neutron interactions. These LETdependent data can be used in conjunction with radiation transport models to calculate dose, dose-equivalent and particle fluxes [7]. For single event effects (SEE) the flux vs. altitude profiles of neutrons, protons and heavy ions are of greatest relevance, although modern devices with very small feature sizes may also be vulnerable to SEU induced by electrons and muons [8]. Figure 4 shows count rates as a function of altitude for three RaySure energy-deposition channels. Channel 2, which primarily measures low LET directly ionizing particles (as well as low energy secondaries from neutron interactions), clearly shows a peak in the 15-20 $\mathrm{km}$ altitude range, reflecting the well-known PfotzerRegener maximum in atmospheric ionization rates [9]. Channel 7, which has a LET threshold approximately one order of magnitude higher, does not show the PfotzerRegener peak, instead plateauing at around $20 \mathrm{~km}$ altitude. By contrast, the RaySure channel with the highest LET threshold, channel 15 , has a near-zero count rate below 20 $\mathrm{km}$ (only one count was recorded below $20 \mathrm{~km}$ ) rising thereafter to approximately 4 counts per hour at the peak altitude of $38 \mathrm{~km}$. This channel requires a minimum energy deposition of $\sim 100 \mathrm{MeV}$ in order to register a count, and thus is not sensitive to direct ionization from protons or electrons. Secondary particles from neutron interactions are also very unlikely to deposit such a large amount of energy in the sensitive volume of the diode, hence this channel's count rate is a reasonable proxy for ion fluxes above a threshold LET of $0.85 \mathrm{MeV} . \mathrm{cm}^{2} / \mathrm{mg}$.

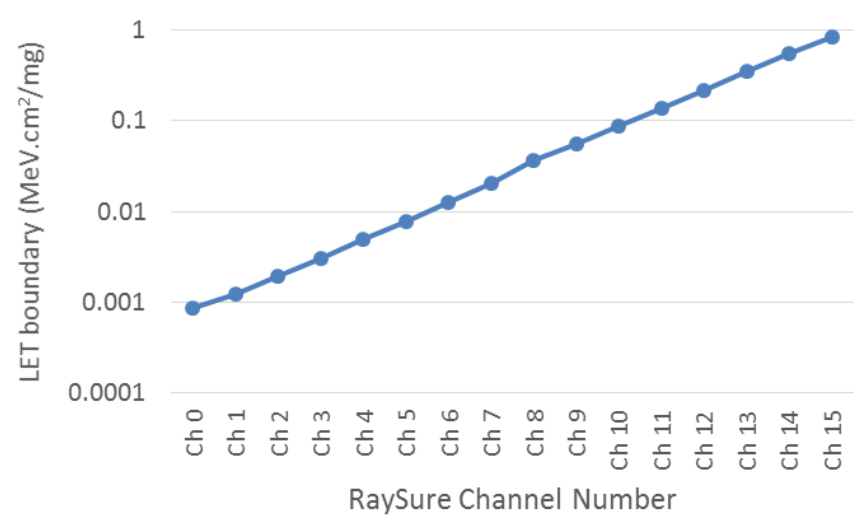

Figure 3: LET thresholds as a function of channel number for the RaySure detector. Thresholds are an approximation based on charge injection under test conditions, with an assumption of normal incidence tracks through the diode's sensitive volume of depth $500 \mu \mathrm{m}$ when converting from charge to LET.

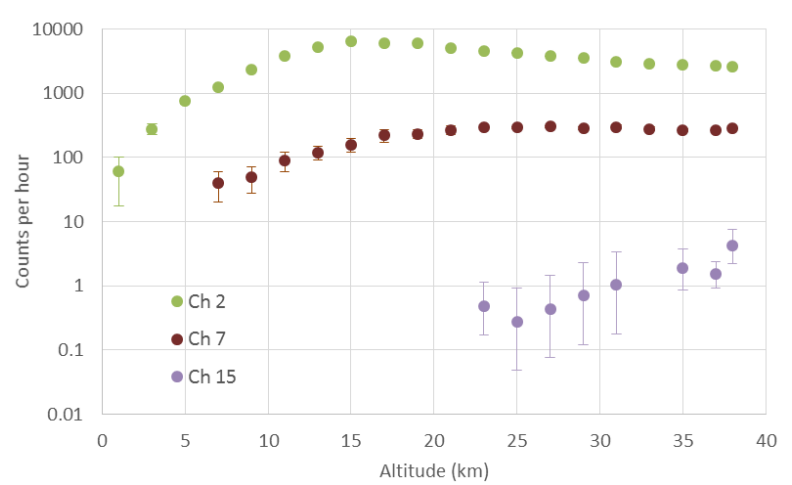

Figure 4: RaySure count rates versus altitude for channels 2, 7 and 15, with LET thresholds of $0.002,0.02$ and $0.85 \mathrm{MeV} . \mathrm{cm}^{2} / \mathrm{mg}$ respectively. The count rate profiles show that the Pfotzer-Regener peak is only observed in the lower energy deposition channels. Error bars are plotted at the $1 \sigma$ level.

It is clear from these data that the prevalence of low LET counts decreases above the Pfotzer-Regener maximum. Figure 5 shows the percentage of the total count rate contributed by the high LET channels (11-15) of RaySure. Below $15 \mathrm{~km}$ this percentage is negligible. Above $15 \mathrm{~km}$ the percentage rises monotonically to its maximum measured value of $\sim 0.36 \%$ at $38 \mathrm{~km}$. There is every expectation that this percentage would continue to rise at even higher altitudes, to an unknown plateau representative of the instrument's response to the primary cosmic ray environment. 


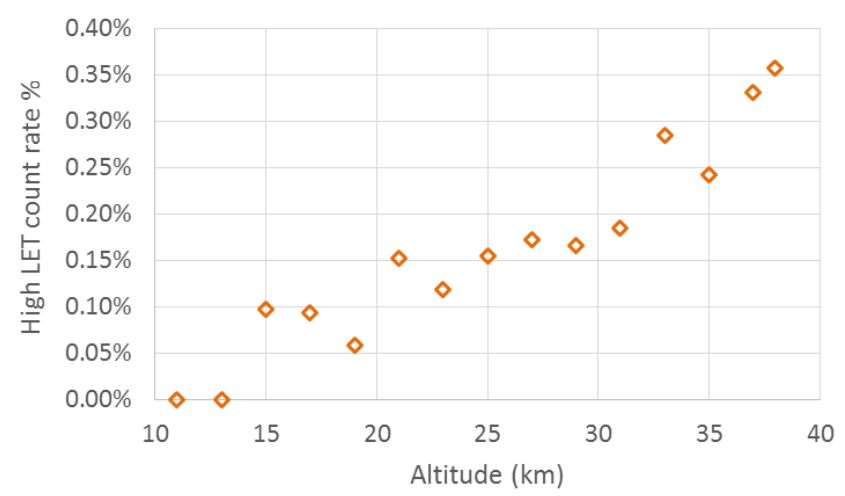

Figure 5: The percentage of the total count rate from RaySure channels 1115 (high LET).

\section{MODELS}

Count rates measured in the lower energy-deposition channels are predominantly caused by secondary particles, especially electrons [7]. Figure 6 shows how electron flux and RaySure count rate (in channel 2) vary with altitude. Electron flux was calculated with the new Model of Atmospheric Ionizing Radiation and Effects (MAIRE) [10]. MAIRE is a parametric model based on particle transport calculations in a multi-layered geometry of the atmosphere using the FLUKA ("FLUktuierende KAskade") Monte Carlo code [11]. The model can, using incident GCR or SPE proton and heavy ion input spectra, calculate fluxes of all relevant particle species, including electromagnetic showers (electrons and photons) and heavy ions (both primaries and secondary fragments), up to an altitude of $100 \mathrm{~km}$.

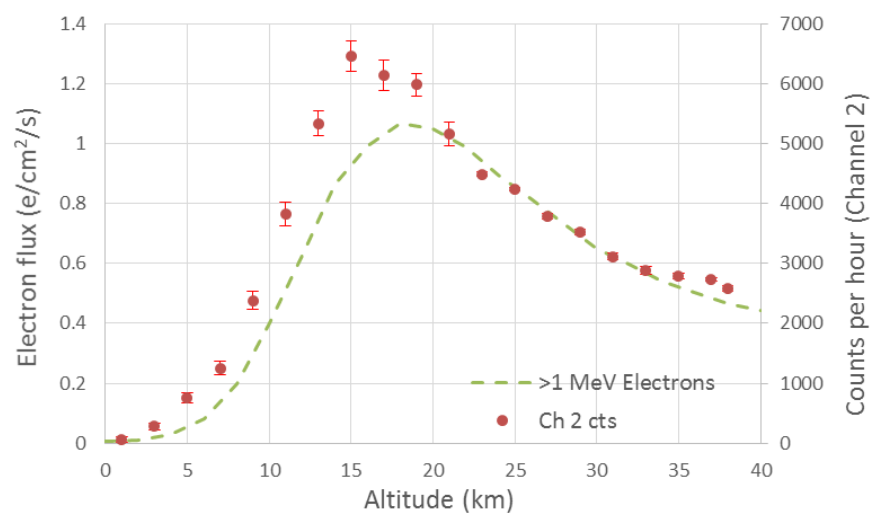

Figure 6: A comparison of the altitude profiles of $>1 \mathrm{MeV}$ electron flux and the count rate of RaySure channel 2. Error bars are plotted at the $1 \sigma$ level.

The altitude profiles of electrons and low LET counts are clearly similar. The measured count rate peaks at a slightly lower altitude than the simulated electron flux (15 km cf. 18 $\mathrm{km})$. However, error bars show that the statistical uncertainty is sufficient to make these results mutually consistent. The cross-sectional area of the diode is approximately $5 \mathrm{~cm}^{2}$, thus an electron flux of $1 \mathrm{e} / \mathrm{cm}^{2} / \mathrm{s}$ implies 18,000 >1 MeV electrons per hour impinging on an area the same size as the RaySure diode, very close to the 20,000 counts per hour accumulated over the low LET channels (where electrons are the dominant contributor [7]). This is, of course, a simplification. Various factors complicate a direct comparison between count rates and particle fluxes: shielding (diode housing, detector structure and wider payload geometry), particle species (particularly for the electron-gamma radiation equilibrium of an electromagnetic shower), path length in the diode, etc. At higher altitudes, anisotropy effects may also come into play as the flux of secondary (as well as primary) particles is biased downward. Unfortunately we do not have sufficient statistics to repeat the analysis in Hands et al. [7] (comparing measured count rates to simulated contributions from each particle species - see Figure 8 in [7]) at each altitude step. Nonetheless, we believe the comparison in Figure 6 corroborates the hypothesis that the lower energy deposition channels are dominated by counts from secondary electromagnetic showers over the full altitude range.

Count rates measured in the higher energy deposition channels below the Pfotzer-Regener maximum are due in large part to indirect ionization due to nuclear interactions between secondary neutrons and silicon nuclei in the diode lattice. However, the profiles in Figure 4 show that these high LET count rates do not peak at $18 \mathrm{~km}$, rather they plateau or even, as in the highest channel, continue to increase with altitude (though statistics are poor in this channel). Figure 7 shows the full set of sixteen RaySure channel count rates as a function of altitude. Also plotted for comparison are neutron and protons fluxes (above $10 \mathrm{MeV}$ ) as a function of altitude (flux is scaled upwards by a factor of 1000 for visualization).

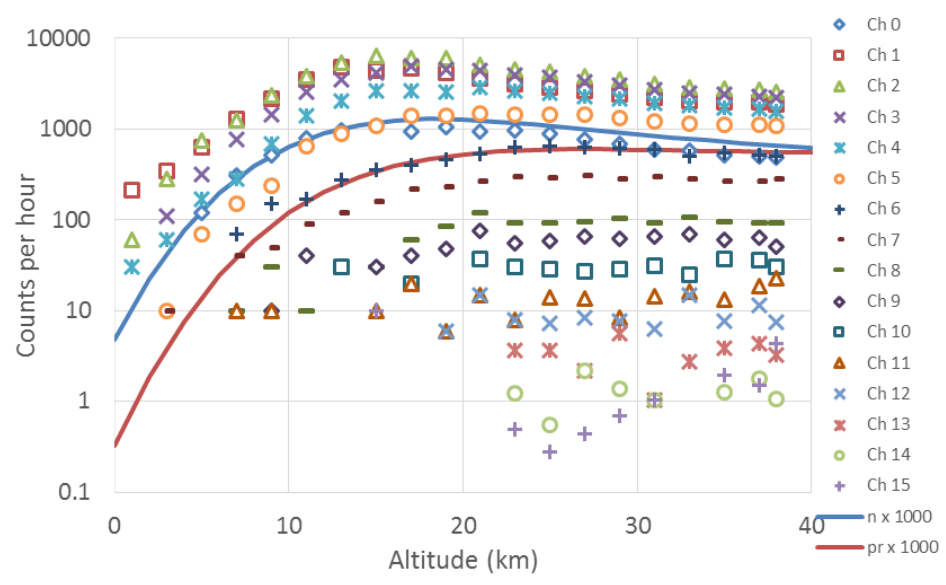

Figure 7: RaySure count rates vs altitude for all sixteen channels. Integral $\left(\# / \mathrm{cm}^{2} / \mathrm{s} />10 \mathrm{MeV}\right)$ neutron and proton fluxes are also plotted for comparison (scaled by a factor of 1000).

The neutron flux vs altitude profile is quite similar to the profile of low energy deposition channels. However, this is somewhat misleading as the interactions between high energy neutrons and silicon nuclei in the detector primarily lead to counts in the higher LET channels [7]. Therefore we are left with the puzzle of increasing (or plateauing) count rates in the higher energy channels, coinciding with decreasing neutron fluxes above the Pfotzer-Regener maximum. The solution must lie with primary cosmic ray particles. Cosmic rays are comprised of protons (85\%), alpha particles or helium nuclei (14\%) and heavier ions $(\sim 1 \%)$. As shown in Figure 8, the elemental composition is slightly different to the solar system as a whole, but the peaks in more stable nuclei such as carbon, oxygen, silicon and iron 
persist. The GCR abundances for $\mathrm{H}$ and $\mathrm{He}$ are for solar minimum at $170 \mathrm{MeV} /$ nucleon, derived from measurements on the IMP-8 spacecraft. The Solar System abundances in this figure are from the compilation by Lodders et al. [12].

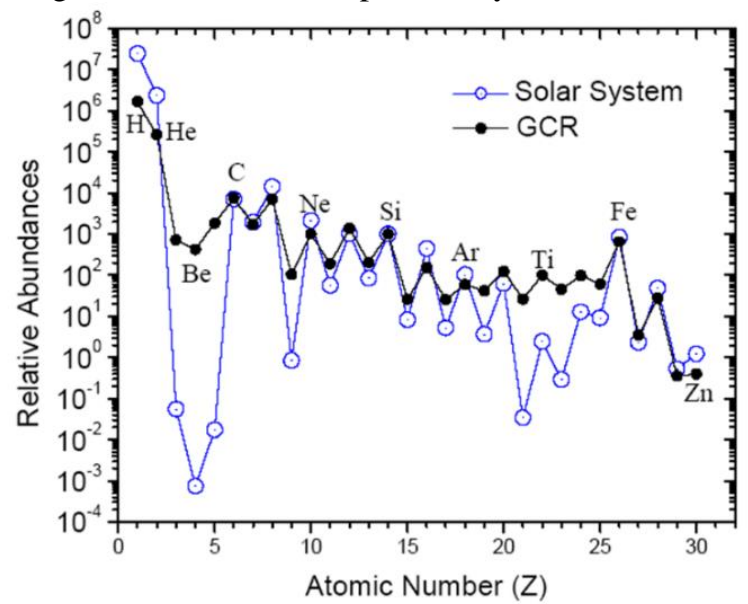

Figure 8: Relative elemental abundances in cosmic rays and the solar system [12].

To establish the flux of primary cosmic ray particles in the atmosphere we used the EXPACS (Excel-based Program for Calculating Atmospheric Cosmic-ray Spectrum) tool [13]. EXPACS is a spreadsheet based tool that provides fluxes of ions up to $\mathrm{Z}=28$ (nickel) in the atmosphere up to an altitude of approximately $60 \mathrm{~km}$ using the PHITS (Particle and Heavy Ion Transport code System) Monte Carlo code [14]. Heavy ions are rapidly attenuated by both the atmosphere and any shielding around the RaySure detector. Hence we are only interested in the most energetic particles and the higher the atomic number, the higher the energy required for a given range. Ion fluxes from EXPACS include both primary and secondary particles in the atmosphere. At lower altitudes the total flux of any given ion species is dominated by low energy secondary ions, whereas at higher altitudes more energetic primary cosmic-ray ions begin to dominate. Figure 9 shows this dual population clearly with fluxes of two types of ion species (helium and carbon) at two altitudes (12 $\mathrm{km}$ and $38 \mathrm{~km})$.

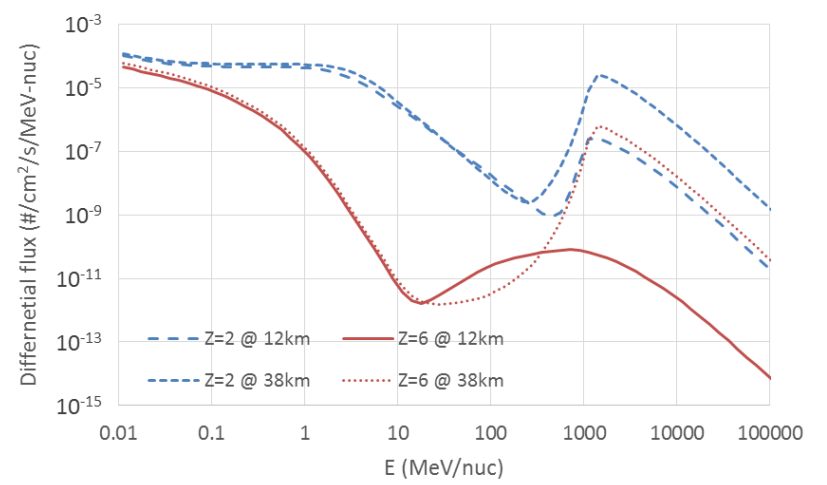

Figure 9: Differential flux spectra of helium $(Z=2)$ and carbon $(Z=6)$ ions at two representative altitudes in the atmosphere. Ion fluxes were calculated with the EXPACS tool using a rigidity cutoff value of $4 \mathrm{GV}$.

The primary cosmic ray particles are clearly visible in the high energy peaks in the spectra in Figure 9. However, there is are significant differences between the two ion species. Energetic alpha particles (helium nuclei) are attenuated by approximately two orders of magnitude between $38 \mathrm{~km}$ and $12 \mathrm{~km}$ (representing the maximum RaD-X altitude and a typical aircraft cruising altitude respectively). The equivalent value for carbon ions is closer to four orders of magnitude, due to the higher LET and thus shorter ranger of higher $\mathrm{Z}$ ions. It is interesting to note that the flux of primary carbon ions at $38 \mathrm{~km}$ is greater than the flux of primary helium ions at $12 \mathrm{~km}$. However, as we are interested in whether cosmic ray ions can contribute to RaySure count rates via direct ionization, it is crucial to consider the LET of each species as a function of energy. LET values calculated using the SRIM code [15] are plotted for helium ions, carbon ions and protons in Figure 10.

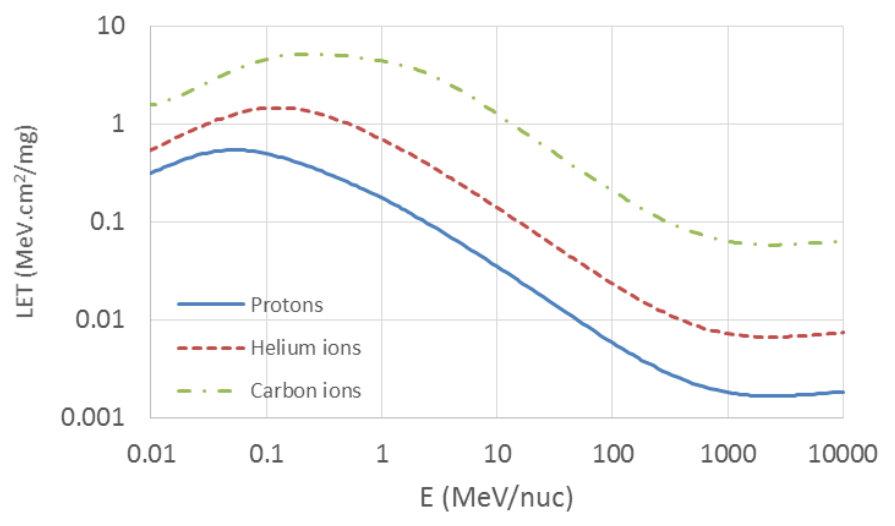

Figure 10: LET of protons, helium ions and carbon ions in silicon, calculated using the SRIM code [15].

From Figure 3 we see that in order to deposit enough energy to register a count in the high LET RaySure channels, a LET of $>0.1 \mathrm{MeV} . \mathrm{cm}^{2} / \mathrm{mg}$ is required (at least for the majority of cases where the path length is of the order of $500-1000$ microns), together with a sufficient initial energy to traverse the detector. Although in an isotropic field the mean path length in a planar sensitive volume is double its thickness, primary cosmic ray flux has a downward bias and thus, for simplicity, in our calculations we use the minimum path length of 500 microns. Figure 11 plots range against energy for the same three ion species. It is clear from these data that protons are unable to induce counts in the higher energy channels via direct ionization. This is because high energy protons fail to meet the minimum energy deposition threshold due to insufficient LET (for example, >17 MeV energy deposition is required for the resultant voltage pulse to be counted in channel 11, which corresponds to a LET threshold of $\sim 0.14 \mathrm{MeV} . \mathrm{cm}^{2} / \mathrm{mg}$ ). By contrast, although Figure 10 shows that low energy protons nominally meet this secondary LET criterion, their total kinetic energy is insufficient to meet the energy deposition threshold that triggers a count. Helium ions with an energy (per nucleon) of $\sim 10-20 \mathrm{MeV}$ may just have sufficient energy to register counts in the medium LET channels, but not in the high LET channels. In any case, these would be secondary ions that do not increase significantly between 12 and $38 \mathrm{~km}$. Carbon ions, by contrast, can meet both the LET and range criteria to deposit counts in the top channels. These ions would need to be of the order of $10 \mathrm{~s}-100 \mathrm{~s} \mathrm{MeV}$, thus according to Figure 9 they fall in the low flux region between primary and secondary particles. 


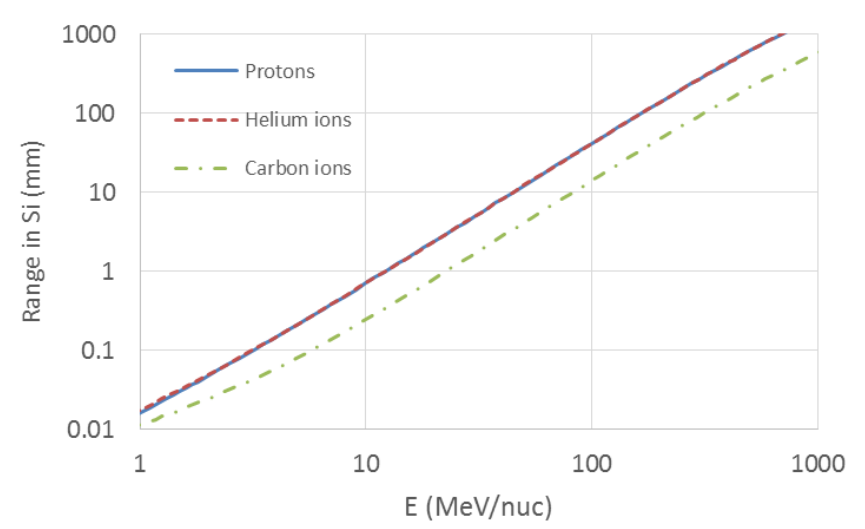

Figure 11: The range in silicon of protons, helium ions and carbon ions. Calculations were performed with the SRIM code [15] and in conjunction with Figure 10 these values demonstrate how high LET particle will not necessarily have sufficient range to register counts in the top RaySure channels.

The rigidity cutoff of $4 \mathrm{GV}$ effectively means that in order for primary cosmic ray ions to induce counts in the higher energy RaySure channels, they must have energies of several $\mathrm{GeV} /$ nuc and thus be close to their minimum ionizing level. As minimum LET scales as the square of the atomic number, it is relatively straightforward to establish the minimum $\mathrm{Z}$ value that is required to induce a count in each of the top channels. These values are given in Table 1.

TABLE 1

ESTIMATE OF THE MINIMUM Z OF IONS THAT ARE ABLE TO TRIGGER COUNTS IN RAYSURE CHANNELS VIA DIRECT IONIZATION.

\begin{tabular}{llll}
\hline Channel & $\begin{array}{l}\text { Min Edep } \\
(\mathrm{MeV})\end{array}$ & $\begin{array}{l}\text { Min LET* } \\
\left(\mathrm{MeV} \cdot \mathrm{cm}^{2} / \mathrm{mg}\right)\end{array}$ & $\begin{array}{l}\text { Min required Z** } \\
\text { (approx. }\end{array}$ \\
\hline 11 & 16.1 & 0.14 & $10(\mathrm{Ne})$ \\
12 & 25.5 & 0.22 & $12(\mathrm{Mg})$ \\
13 & 41.0 & 0.35 & $15(\mathrm{P})$ \\
14 & 64.4 & 0.55 & $19(\mathrm{~K})$ \\
15 & 98.8 & 0.85 & $24(\mathrm{Cr})$ \\
\hline
\end{tabular}

*For minimum path length of $500 \mu \mathrm{m}$

**Assuming minimum ionizing particles

Now that we have an idea which ion species could feasibly cause counts in the higher RaySure channels, we can use EXPACS to establish the flux vs altitude profile of the relevant ion species. These profiles are shown in Figure 12 using $1000 \mathrm{MeV} /$ nucleon as the integral flux energy threshold and an input geomagnetic cutoff rigidity of $4 \mathrm{GV}$. Several features stand out from these plots. The fluxes of each species increase rapidly with altitude between 10 and $20 \mathrm{~km}$, implying that their influence at aircraft altitudes is negligible (consistent with Figure 5). Above $20 \mathrm{~km}$ the increase with altitude continues at a lower gradient, and the flux ratio between species decreases (approaching the abundance ratios of the primary cosmic ray population). Qualitatively, these flux vs altitude profiles appear to be considerably steeper than the higher channel profiles in Figure 7 (which, apart from channel 15, are broadly flat). The count rates of these channels $(11-15)$ at high altitudes are in the range 1-10 counts per hour. A naïve calculation, again using the cross-section of the diode, implies a required total particle flux in the range $5 \times 10^{-5}-5 \times 10^{-4}$ ions $/ \mathrm{cm}^{2} / \mathrm{s}$, if these counts are caused by direct ionization. The data plotted in Figure 12 imply that, the respective fluxes of ion species, at the minimum required $\mathrm{Z}$ value for each channel, are lower than this range. However, total flux of particles capable of inducing counts in each channel is larger than this crude measure, and complicated by the fluctuating abundance ratios in Figure 8, where higher $\mathrm{Z}$ species can actually have higher fluxes.
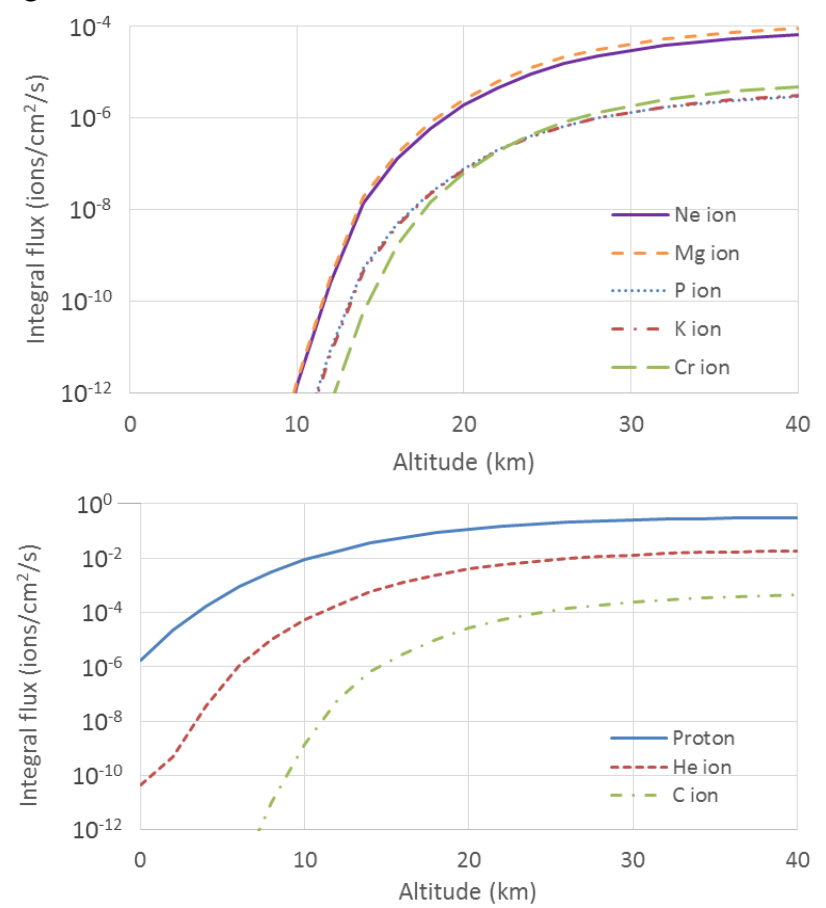

Figure 12: Integral flux of high energy ( $>1000 \mathrm{MeV} /$ nuc) primary cosmic ray ions as a function of altitude in the atmosphere at a geomagnetic cutoff rigidity of $4 \mathrm{GV}$, calculated with EXPACS. The top panel plots ion species from Table 1, the bottom panel plots fluxes for lower $Z$ species for reference.

In order to compare RaySure count rates to cumulative ion fluxes across the different species, it is instructive to calculate aggregate LET spectra. MAIRE is capable of outputting ion fluxes as a function of LET at any point in the atmosphere. This capability can subsequently be used to calculate single event upset (SEU) rates using an integrated tool based on the IRPP method [16], this is discussed in the next section. Figure 13 shows three examples of atmospheric LET spectra, at 0,12 and $38 \mathrm{~km}$ altitude (representing ground level, commercial aviation and the $\mathrm{RaD}-\mathrm{X}$ peak altitude respectively) and at a cut-off rigidity of $4 \mathrm{GV}$ (Fort Sumner). These results show that, although at low LET the high altitude environment is similar to that at aviation altitudes, at higher LET values the ion flux is significantly greater. This difference reflects the high level of attenuation of cosmic ray ions in the layers of the atmosphere above $12 \mathrm{~km}$. In addition, it is evident from Figure 9 that the difference in high altitude, high LET flux is due to primary cosmic rays rather than secondary low energy (but high LET) ions. 


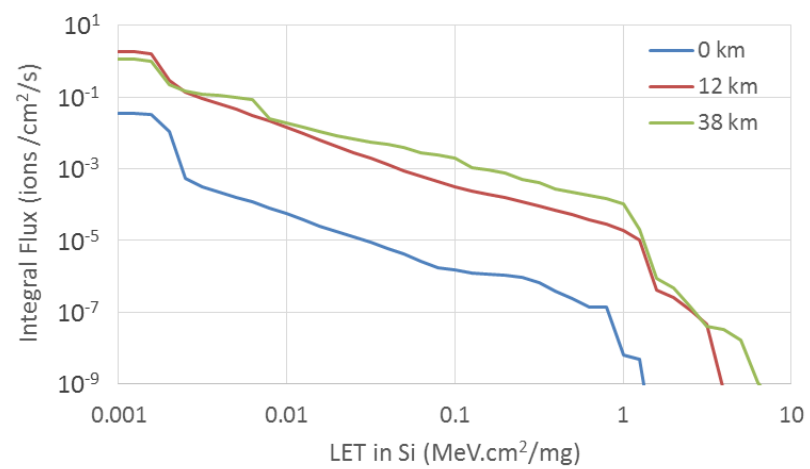

Figure 13: Integral ion flux spectra as a function of LET, produced by the MAIRE model at three different altitudes. These data are for a geomagnetic cut-off rigidity of $4 \mathrm{GV}$, representing the $\mathrm{RaD}-\mathrm{X}$ launch site.

We use these data to estimate count rates based on LET ranges, using the data in Table 1 as our guide. Figure 14 compares output from the MAIRE model to RaySure data from the RaD-X flight. Upper and lower plots show respectively the measured RaySure channel 11 and channel 15 count rates compared to a predicted rate calculated by multiplying ion fluxes in appropriate LET ranges by the active area of the detector diode. This is clearly an approximation as ions outside the given LET ranges can also induce counts in each channel, both with longer path lengths at oblique angles, or shorter path lengths at diode edges. A further complication is the influence of detector shielding on ion fluxes in the sensitive volume. The effect of this additional shielding on estimated count rates is expected to be relatively small, perhaps equivalent to a reduction in altitude of one or two km. As shown in Figure 14, this would not have a significant effect on the comparison with measurements.
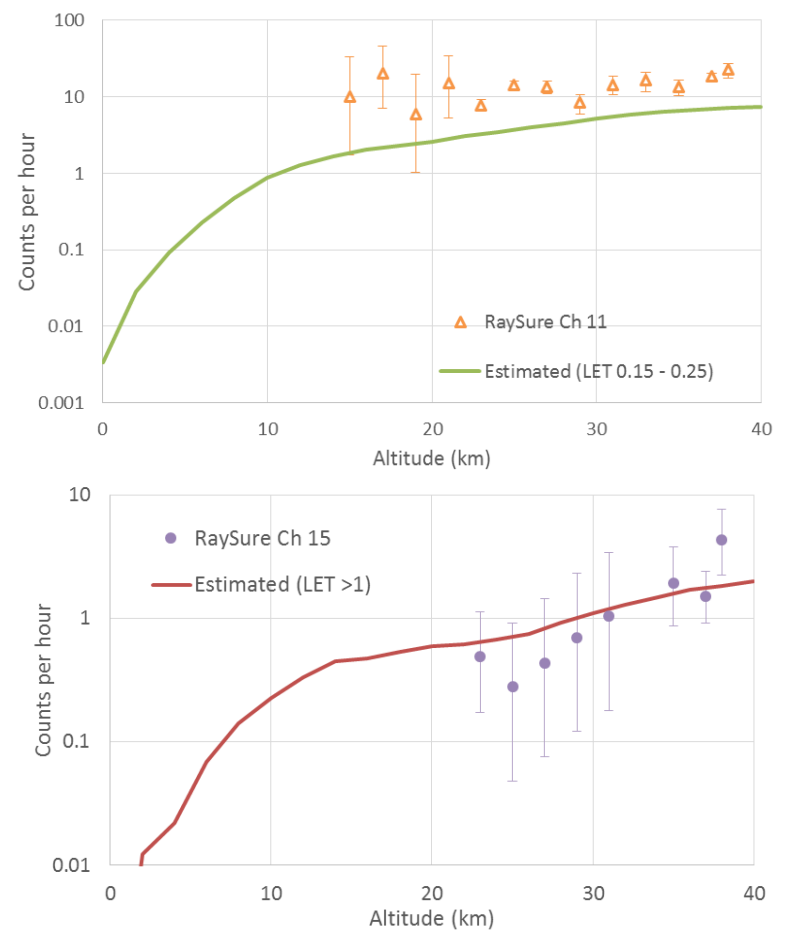

Figure 14: (Top) RaySure channel 11 count rate compared to predicted count rate based on $0.25>\mathrm{LET}>0.15 \mathrm{MeV} . \mathrm{cm}^{2} / \mathrm{mg}$ flux multiplied by PIN diode cross-sectional area. (Bottom) RaySure channel 15 count rate compared to predicted count rate based on LET $>1 \mathrm{MeV} . \mathrm{cm}^{2} / \mathrm{mg}$ flux multiplied by PIN diode cross-sectional area.
These comparisons show that, within the counting statistics of the data, the count rate in the highest RaySure channel can be accounted for entirely by direct ionization from heavy ions. In actual fact, of course, indirect ionization in this channel is also possible, and this will represent a fraction of the count rate. Within the statistics of our data, we cannot establish what this fraction is. The picture for channel 11 is slightly different. Here our crude estimation of count rate from direct ionization is lower than the measured count rate, at least for altitudes above $20 \mathrm{~km}$. Even in this channel the statistical errors are significant, but this method implies that the majority of counts $(\sim 50-80 \%)$ are caused by indirect ionization. As the neutron flux is diminishing, it must be primary cosmic ray protons that are predominantly responsible. This conclusion is consistent with the qualitative observation that the gradient of primary cosmic ray ion flux is lowest for protons (Figure 12), mimicking the very low gradients of the higher LET channels (excepting channel 15).

This comparison between model-based ion fluxes, LET spectra and empirical count rates is constrained by statistical errors on the data, and the presence (possibly dominant) of counts from indirect ionization in the higher LET RaySure channels. Nonetheless, we believe that the both the qualitative and quantitative comparisons we have made, give confidence to the fidelity of the models used. We now go on to exploit these models for SEE calculations in the atmosphere.

\section{Single EVEnT UPSETS}

At aviation altitudes SEE rates are assumed to be dominated by neutron-induced events [17]. However, at higher altitudes direct ionization from ions inevitably become an increasingly dominant influence. This could be accentuated by the sensitivity of some modern (and future) devices to SEE from proton direct ionization [18]. MAIRE can calculate SEU rates from indirect ionization due to protons and neutrons using particle fluxes and a standard bit upset cross-section calculation [19]. In addition, using the LET spectra shown earlier, the model can also calculate SEU rates from ions using the IRPP method [16]. In this method, the integral LET spectrum, combined with the path-length distribution through a parallelepiped representation of a device sensitive volume, will yield the expected SEU rate for the device. For such calculations it is necessary to have parametric test data for specific memory devices. Two 4Mbit SRAM, from Hitachi and Samsung respectively, have been fully characterized using heavy ion, proton and neutron beams [20] [21] [22] and the cross-section data are summarized in Table 2 . The device cross section vs LET is expressed as a Weibull function, $\sigma=\sigma_{0}\left\{1-\exp \left(-\left[\left(\mathrm{L}-\mathrm{L}_{0}\right) / \mathrm{W}\right]^{\mathrm{s}}\right)\right\}$. The sensitive volume of the Hitachi SRAM is estimated to be $4.5 \times 4.5 \times 0.5 \mu^{3}$ and the Samsung SRAM is estimated at $0.5 \times 0.5 \times 0.5 \mu \mathrm{m}^{3}$. These dimensions, whilst not at the same feature size of the most recent nanometric-scale devices, can be thought of as representative of devices currently in use in avionics systems. Conveniently, the very different sensitive volume shapes (approximately planar and cubic), capture the 
very significant effect of path length distribution on heavy ion SEE calculation with IRPP.

TABLE 2

WEIBULL SEU CROSS-SECTIONS PARAMETERS OF THE HITACHI (PART NO. HM628512ALP-7) AND SAMSUNG (PART NO. KM684002AJ-17) 4-MBIT SRAMS. THE DATA BEHIND THESE FITS ARE PRESENTED IN [20], [21] AND [22].

\begin{tabular}{|c|c|c|c|c|c|}
\hline \multirow{2}{*}{$\begin{array}{l}\text { Device } \\
\text { Label }\end{array}$} & \multirow[t]{2}{*}{ Particle } & \multicolumn{4}{|c|}{ Weibull Parameters (and units) } \\
\hline & & $\begin{array}{l}\mathrm{L}_{0} / \mathrm{E}_{0} \\
\mathrm{MeV} \cdot \mathrm{cm} 2 / \mathrm{mg}\end{array}$ & $\begin{array}{l}\mathrm{W} \mathrm{MeV} \text { or } \\
\mathrm{MeV} \cdot \mathrm{cm} 2 / \mathrm{mg}\end{array}$ & $\mathrm{s}$ & $\begin{array}{l}\sigma_{0} \\
\mathrm{~cm} 2 / \text { bit }\end{array}$ \\
\hline \multirow[t]{3}{*}{ Hit A } & Ions & 3.1 & 21.68 & 1.24 & $2.5 \times 10^{-7}$ \\
\hline & Protons & 12.65 & 11.8 & 2.19 & $6.7 \times 10^{-14}$ \\
\hline & Neutrons & 2.5 & 14.87 & 2.03 & $1.2 \times 10^{-13}$ \\
\hline \multirow[t]{3}{*}{ Sam A } & Ions & 1 & 139.6 & 1.6 & $2.5 \times 10^{-9}$ \\
\hline & Protons & 2.5 & 17.4 & 1.51 & $2.0 \times 10^{-16}$ \\
\hline & Neutrons & 2.5 & 17.4 & 1.51 & $2.0 \times 10^{-16}$ \\
\hline
\end{tabular}
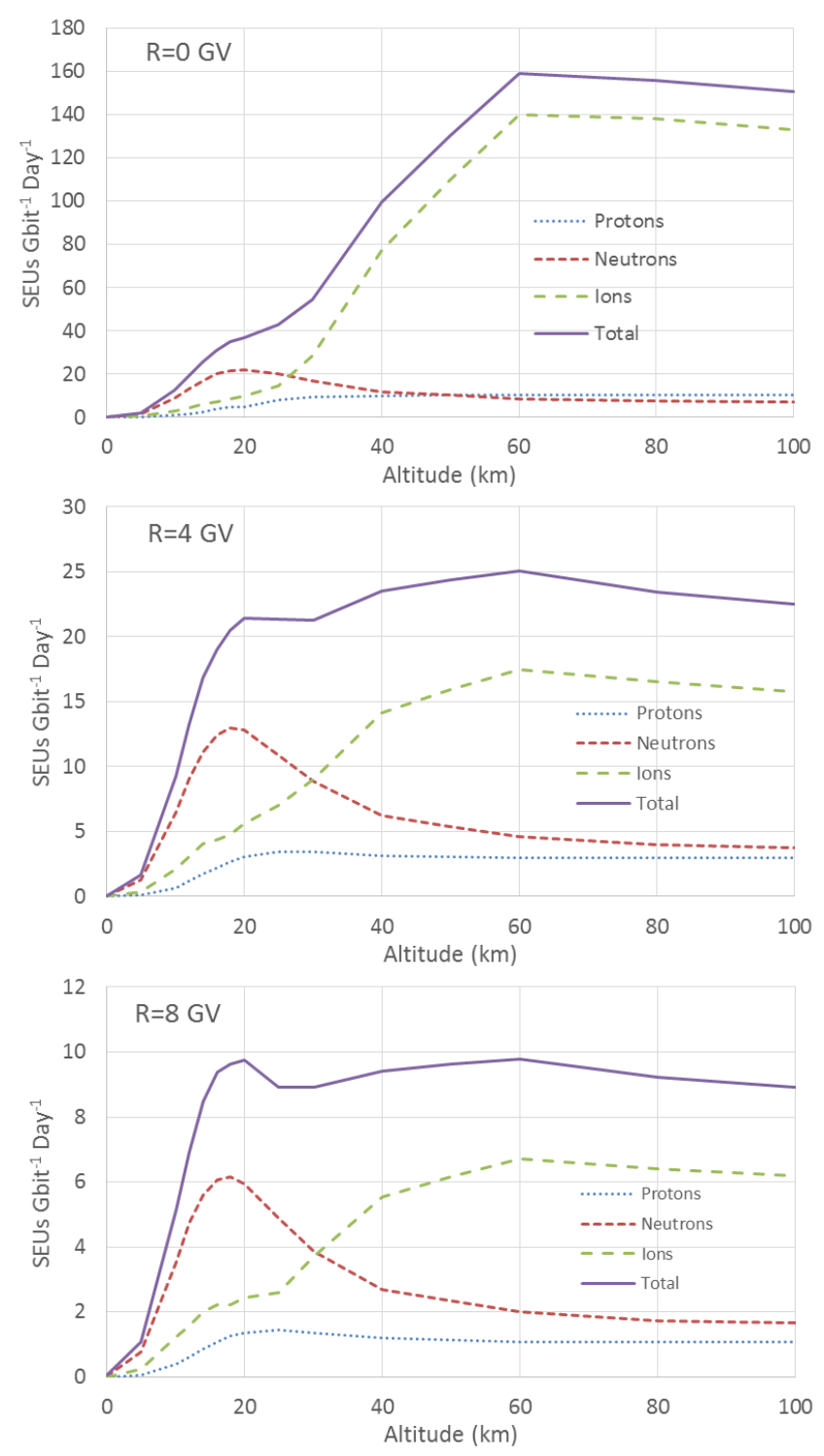

Figure 15: Calculations of SEU rates vs. altitude for protons, neutrons and ions at three cut-off rigidities for a Hitachi SRAM.

We have used MAIRE with these parameters to calculate SEU rates in the atmosphere from ground level up to $100 \mathrm{~km}$ (thus far exceeding the $\mathrm{RaD}-\mathrm{X}$ altitude range). In addition to the $4 \mathrm{GV}$ geomagnetic cutoff rigidity of $\mathrm{RaD}-\mathrm{X}$, we have repeated these calculations for two further cutoffs: 0 and 8
GV. $0 \mathrm{GV}$ represents the worst case (no geomagnetic shielding) and $8 \mathrm{GV}$ represents a more conservative case with a consistent step change in rigidity cutoff. Figure 15 shows predictions of SEU rates as a function of altitude at three cut-off rigidities for the Hitachi device. Figure 16 shows predictions of SEU rates as a function of altitude at three cut-off rigidities for the Samsung device.
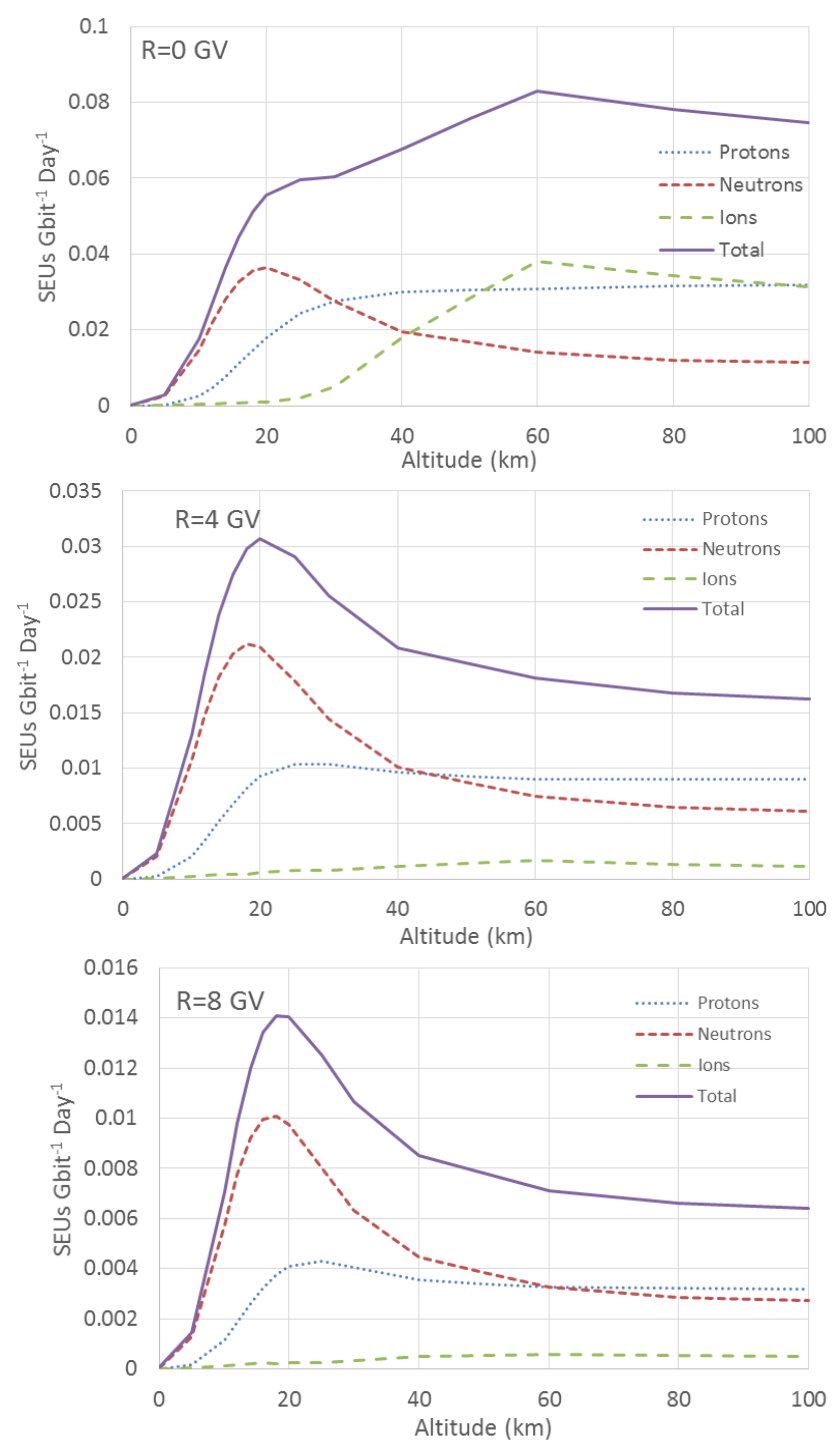

Figure 16: Calculations of SEU rates vs. altitude for protons, neutrons and ions at three cut-off rigidities for a Samsung SRAM.

Various conclusions can be inferred from the structure of these SEU vs altitude plots. The most striking is the much higher absolute SEU rates for the Hitachi device at all three cutoff rigidities. This is, of course, due to the much larger sensitive volume of the Hitachi device. For direct ionization (heavy ion) SEU the planar volume allows for much longer path lengths and thus greater energy deposition. The IRPP rate calculation is complex convolution of flux as a function of LET, and the path length distribution within the device sensitive volume. Therefore the increase is not a simple linear scaling (e.g. the ratio of heavy ion SEU rate between the devices at $60 \mathrm{~km}$ and $0 \mathrm{GV}$ is $\sim 3500$, whereas the sensitive volume ratio is 81 ). Even for indirect ionization SEU (protons and neutrons) the ratios are, respectively, $\sim 330$ 
and $\sim 600$, i.e. again significantly larger than the volume ratio. This is because in the Samsung device the secondary products from nuclear reactions (primarily protons, alpha particles and $\mathrm{Si}$ nuclei) are more likely to 'escape' the sensitive volume before depositing all their energy.

At all three rigidity cutoffs the cubic volume of the Samsung device restricts the contribution of heavy ion SEU relative to neutron and proton SEU. This leads to a well-defined peak in the SEU rate in all but the zero rigidity case, coincident with the Pfotzer-Regener peak in ionization rate. The dominance of heavy ion induced SEU in the Hitachi device, with a more planar sensitive volume, has the effect of washing out all but a hint of the Pfotzer-Regener maximum. Clearly, determining whether the stratospheric environment becomes more or less hostile in terms of SEU will depend very much on the device geometry, as this affects the ratio between direct ionization SEU and indirect ionization SEU. The apparent dip in heavy ion SEU rates above $60 \mathrm{~km}$, which is common to all profiles, is due to the flux of secondary ions (which is lower at the top of the atmosphere). The LET thresholds of the devices (3.1 and $1 \mathrm{MeV} . \mathrm{cm}^{2} / \mathrm{mg}$ respectively) mean that they are less susceptible to SEU from primary minimum-ionizing ions (either a high $\mathrm{Z}$ or long path length is required). However, these ions lose energy in the very low density upper atmosphere (mesosphere), thus increasing their LET without being absorbed. Low energy (high LET) ions are also produced by spallation reactions between primary cosmic rays and the atmosphere. This results in an increasing flux of high LET ions at mesospheric altitudes $(>50 \mathrm{~km})$, before the rapid drop off when they are absorbed in the stratosphere. Shielding from the packaging of a sensitive electronic component (or its outer housing) will also influence the location of this peak in the high LET flux. Hence the ion SEU vs altitude profiles in Figure 15 and Figure 16, which do not assume any shielding from device packaging, may be slightly different in practice. This, however, does not affect the overall trend of the increasing dominance of heavy ion SEU with altitude.

\section{DISCUSSION}

In this work we have considered SEU from protons, neutrons (via nuclear interactions) and ions (via direct ionization). These are the primary sources of SEU in the atmospheric environment. Other possible sources of SEU, such as direct ionization from protons [23] [24], electroninduced SEU [25], and muon-induced SEU [26], have been excluded from this analysis. This is in part because the feature sizes of the devices we have used for our case study (hundreds of $\mathrm{nm}$ ) are too large for these secondary effects to be viable. However, even for devices with feature sizes an order of magnitude lower, we believe the dominance of secondary neutron flux at lower altitudes, and primary proton flux at higher altitudes is likely to persist, though it has been suggested that electron-induced SEU could dominate in devices with extremely low critical charge thresholds [8]. As feature sizes continue to shrink, this hypothesis should ideally be tested with direct in-situ measurements of SEU on high altitude platforms. We aim to expand our investigations in this area by deploying solid-state detectors (such as
RaySure) alongside bespoke test boards for measuring SEU and other types of SEE. Only in this way can the link between environmental measurements and error rates be properly established. In addition, the separation of error rates into constituent elements from different particle species will require a complementary campaign of ground experiments, where the disentanglement problems inherent to a mixed-field radiation environment can be avoided.

At present there is a large gap in maturity between radiation effects research in the space community and equivalent activity in the aviation community. Although SEE in avionics has been known about for some time [27], the development of international standards to mitigate these effects is more recent [28], [29] and thus less established than equivalent design standards for space-based platforms. With the increasing ubiquity of fly-by-wire avionics systems, and the likely expansion to higher altitude platforms, it is imperative that this gap is narrowed and this work is intended as a first step in that process.

\section{CONCLUSION}

We have used empirical data from a high altitude balloon flight to validate the output of a new comprehensive atmospheric radiation model, MAIRE. This model has been used to calculate SEU rates as a function of altitude in the atmosphere using two representative SRAM devices. These examples show that at altitudes above those used by commercial aviation, the influence of penetrating cosmic ray heavy ions becomes the dominant factor causing SEU (and thus other SEE), with rates at 50-100 km up to ten times those at $10-12 \mathrm{~km}$. These findings are of great significance for the reliability of avionics in suborbital space-tourism aircraft and other high altitude aircraft, UAVs and balloons.

\section{ACKNOWLEDGMENT}

This work was funded by the NASA Science Mission Directorate under the Hands-On Project Experience (HOPE)-4 opportunity. The authors would like to express our gratitude to the whole $\mathrm{RaD}-\mathrm{X}$ team at NASA Langley Research Center, who guided the RaD-X project to success and supported the inclusion of RaySure on the payload throughout. We are also very grateful to all the staff at the Columbia Scientific Balloon Facility in Fort Sumner.

\section{REFERENCES}

[1] C. H. Tsao, R. Siberberg, J. H. Adams and J. Letaw, "Cosmicray transport in the atmosphere: Dose and LET-distribution in materials," IEEE Trans. Nucl. Sci., Vols. NS-30, no. 6, pp. 4398-4404, 1983.

[2] L. Artola, R. Velazco, G. Hubert, S. Duzellier, T. Nuns, B. Guerard, P. Peronnard, W. Mansour, F. Pancher and F. Bezerra, "In Flight SEU/MCU Sensitivity of Commercial Nanometric SRAMs: Operational Estimations," IEEE Trans. Nucl. Sci., vol. 58, no. 6, pp. 2644 - 2651, 2011.

[3] F. Wrobel, J. Vaille, D. Pantel, L. Dilillo, P. Rech, J.-M. Galliere, A. Touboul, P. Chadoutaud, P. Cocquerez, M. 
Lacourty, T. Lam-Trong, J.-L. Autran, C. Chatry, F. Laplanche, B. Azais and F. Saigne, "Experimental Characterization of an Atmospheric Environment With a Stratospheric Balloon," IEEE Trans. Nucl. Sci., vol. 58, no. 3, pp. 945 - 951, 2011.

[4] G. Hubert, F. Bezerra, J. -M. Nicot, L. Artola, A. Cheminet, J. -N. Valdivia, J. -M. Mouret, J. -R. Meyer and P. Cocquerez, "Atmospheric Radiation Environment Effects on Electronic Balloon Board Observed During Polar Vortex and Equatorial Operational Campaigns," IEEE Trans. Nucl. Sci., vol. 61, no. 4, pp. 1703 - 1709, 2014.

[5] L. Townsend, M. PourArsalan, F. Cucinotta, M. Kim and N. Schwadron, "Transmission of galactic cosmic rays through Mars atmosphere," Space Weather Journal, vol. 9, no. 6, 2011.

[6] "NASA RaD-X," [Online]. Available: http://radX.larc.nasa.gov/.

[7] A. Hands and C. S. Dyer, "A Technique for Measuring Dose Equivalent and Neutron Fluxes in Radiation Environments Using Silicon Diodes," IEEE Trans. Nucl. Sci., vol. 56, no. 6, pp. 3442-3449, 2009.

[8] J. Barak and N. M. Yitzhak, "SEU Rate in Avionics: From Sea Level to High Altitudes," IEEE Tran. Nucl. Sci., vol. 62, no. 6, pp. 3369-3380, 2015.

[9] F. Wissmann, O. K. S. Burda, T. Klages and F. Langner, "Dosimetry of Secondary Cosmic Radiation up to an Altitude of 30 km," Rad. Prot. Dos., vol. 161, no. 1-4, pp. 299-302, 2014.

[10] "MAIRE," RadMod Research, [Online]. Available: www.radmod.co.uk/maire.

[11] "FLUKA," [Online]. Available: www.fluka.org.

[12] K. Lodders, "Solar System Abundances and Condensation Temperatures of the Elements," The Astrophysical Journal, vol. 591, no. 2, pp. 1220-1247, 2003.

[13] T. Sato, "Analytical Model for Estimating Terrestrial Cosmic Ray Fluxes Nearly Anytime and Anywhere in the World: Extension of PARMA/EXPACS.," PLoS ONE, vol. 10, no. 12, 2015 .

[14] T. Sato, H. Yasudab, K. Niitac, A. Endoa and L. Sihver, "Development of PARMA: PHITS-based Analytical Radiation Model in the Atmosphere," Radiation Research, vol. 170, no. 2, pp. 244-259, 2008.

[15] J. Ziegler, The Stopping and Range of Ions in Matter, Oxford, UK: Pergamon Press, 1997.

[16] E. L. Petersen, "Cross section measurements and upset rate calculations," IEEE Trans. Nucl. Sci., vol. 43, no. 6, pp. 28052819, 1996.

[17] E. Normand, "Correlation of Inflight Neutron Dosimeter and SEU Measurements With Atmospheric Neutron Model," IEEE Trans. Nucl. Sci., vol. 48, no. 6, pp. 1996-2003, 2001.

[18] P. Marshall, K. LaBel, J. Schwank, K. Rodbell, M. Hakey, M. Berg, P. Dodd, M. Friendlich, A. Phan, C. Seidleck, M. Shaneyfelt and M. Xapsos, "Low Energy Proton SingleEvent-Upset Test Results on $65 \mathrm{~nm}$ SOI SRAM," IEEE Trans. Nucl. Sci., vol. 55, no. 6, pp. 3394 - 3400, 2008.

[19] A. Hands, C. Dyer and F. Lei, "SEU Rates in Atmospheric Environments: Variations Due to Cross-Section Fits and Environment Models," IEEE Trans. Nucl. Sci., vol. 56, no. 4, pp. 2026-2034, 2009.

[20] C. S. Dyer, S. N. Clucas, F. Lei, P. Truscott, R. Nartello and C. Comber, "Comparative simulations of single event upsets induced by protons and neutrons in commercial SRAMs," in
Proceedings of RADECS 2003, ESA, Noordwijk, Holland, 2003.

[21] C. Poivey, B. Doucin, M. Bruggemann and R. HarboeSorensen, "Radiation characterisation of commercially available $1 \mathrm{Mbit} / 4 \mathrm{Mbit}$ SRAMs for space applications," in Radiation Effects Data Workshop, NSREC, Newport Beach, California, 1998.

[22] A. Hands, P. Morris, C. Dyer, K. Ryden and P. Truscott, "Single Event Effects in Power MOSFETs and SRAMs Due to $3 \mathrm{MeV}, 14 \mathrm{MeV}$ and Fission Neutrons," IEEE Trans. Nucl. Sci, vol. 58, no. 3, pp. 952 - 959, 2011.

[23] L. D. Edmonds and K. J. Edmonds, "A Method for Estimating SEU Rates From Protons by Direct Ionization," IEEE Trans. Nucl. Sci., vol. 55, no. 5, pp. 2666 - 2678, 2008.

[24] K. P. Rodbell, D. F. Heidel, H. H. K. Tang, M. S. Gordon, P. Oldiges and C. E. Murray, "Low-Energy Proton-Induced Single-Event-Upsets in $65 \mathrm{~nm}$ Node, Silicon-on-Insulator, Latches and Memory Cells," IEEE Trans. Nucl. Sci., vol. 54, no. 6, pp. 2474 - 2479, 2007.

[25] J. M. Trippe, R. A. Reed, R. A. Austin, B. D. Sierawski, R. A. Weller, E. D. Funkhouser, M. P. King, B. Narasimham, B. Bartz, R. Baumann, J. Labello, J. Nichols, R. D. Schrimpf and S. L. Weeden-Wright, "Electron-Induced Single-Event Upsets in $28 \mathrm{~nm}$ and $45 \mathrm{~nm}$ Bulk SRAMs," IEEE Trans. Nucl. Sci., vol. 62, no. 6, pp. 2709-2716, 2015.

[26] B. D. Sierawski, M. H. Mendenhall, R. A. Reed, M. A. Clemens, R. A. Weller, R. D. Schrimpf, E. W. Blackmore, M. Trinczek, B. Hitti, J. A. Pellish, R. C. Baumann, S.-J. Wen, R. Wong and N. Tam, " Muon-Induced Single Event Upsets in Deep-Submicron Technology," IEEE Trans. Nucl. Sci., vol. 57, no. 6, pp. 3273 - 3278, 2010.

[27] C. S. Dyer, A. J. Sims, J. Farren and J. Stephen, "Measurements of the SEU environment in the upper atmosphere," IEEE Trans. Nucl. Sci., vol. 36, no. 6, pp. 2275 - 2280, 1989.

[28] IEC62396-1, "Process management for avionics Atmospheric radiation effects - Part 1: Accommodation of atmospheric radiation effects via single event effects within avionics electronic equipment," International Electrotechnical Commission, 2016.

[29] R. Edwards, C. Dyer and E. Normand, "Technical standard for atmospheric radiation single event effects (SEE) on avionics electronics," in IEEE Radiation Effects Data Workshop, Atlanta, GA, USA, 2004. 